\title{
Time-dependent Infiltration from Periodic Semi- Circular Channels with Two Different Types of Root-Water Uptake
}

\author{
Imam Solekhudin \\ Department of Mathematics, Faculty of \\ Mathematics and Natural Sciences \\ Universitas Gadjah Mada \\ Yogyakarta, Indonesia \\ imams@ugm.ac.id
}

\begin{abstract}
In this study, time dependent infiltration problems from periodic semicircular channels with root-water uptake function are considered. The considered problems are governed by Richards equation. To scrutinize the equation more conveniently, the equation was transformed into a modified Helmholtz equation using a set of transformations involving Laplace transform. A Laplace transform dual reciprocity method (LTDRM) with a predictor-corrector scheme, which was used to solve the modified Helmholtz equation numerically. Using the solution obtained, numerical values of amount of water absorbed by the different plant roots distribution can be computed.
\end{abstract}

Keywords-infiltration, periodic channels, Helmholtz equation, LTDRM

\section{INTRODUCTION}

Problems involving water infiltration in homogeneous soils have been considered by a number of researchers, such as Batu, Clements et al., Solekhudin, Clements and Lobo [15]. Batu considered steady infiltration from flat channels [1]. In this study, Batu gave analytic solutions of steady infiltration from periodic channels and single channel. Clements et al. [2] studied steady infiltration problems with impermeable layers. In this study, influence of the impermeable layers to water flow in soil is investigated. Solekhudin [3,4] investigated steady infiltration from periodic channels with root-water uptake. Time-dependent infiltration from single channel was studied by Clements and Lobo [5]. Location gives an impact to the speed of convergence.

This paper is a continuation of previous research [3,4]. We investigate solutions to a problem involving time dependent infiltration from periodic semi-circular channels with absorption by plant roots in a homogeneous soil. Two different types of root uptake are considered. A set of transformations, involving Laplace transform, was used to convert the governing equation of the problem into a modified Helmholtz equation. To solve the equation numerically, a method involving a DRBEM and a predictorcorrector scheme is employed.

\section{METHODS}

Problem Formulation
This paper is a continuation of what was discussed in Solekhudin [4], hence its formulation is very similar. Using a Cartesian frame $O X Y Z$ with $O Z$ vertically positive downwards, we consider a type of homogeneous soil, pima clay loam (PCL) in the region $Z \geq 0$. On the surface of the soil, Periodic semicircular channels of $2 L$ per unit length surface area are created. Crops, with roots of depth $Z m$ and width $2 X_{m}$, are planted between and equidistant from two adjacent channels. The distance between the centers of two consecutive channels is $2(L+D)$. An illustration of this description is shown in Fig. 1. This illustration is as that in the study reported by Solekhudin and Sumardi [6]. Given this situation, we consider a time-dependent infiltration problem involving infiltration from periodic semi-circular channels with rootwater uptake.

It is assumed that cross section of the channels and the root distribution do not change in the OY direction. We also assume that they are symmetrical about the planes $X=$ $\pm k(L+D)$, for $k=0,1,2, \ldots$ The channels are filled with water. Water fluxes on the channels are uniform, $v_{0}$. The flux on the soil surface outside the channels is zero. Since the geometry of the problem does not change in $O Y$ direction, it is sufficient to consider the semi-infinite region defined by $0 \leq X \leq L+D$ and $Z \geq 0$, which is denoted by $R$ with boundary $C$. The fluxes over the surface of the channels and over the surface of soil outside the channels are $v_{0}$ and 0 respectively. Since the problem are symmetrical about $X=0$ and $X=L+D$, there are no fluxes across them. The derivatives $\partial \Theta / \partial X \rightarrow 0$ and $\partial \Theta / \partial Z$ $\rightarrow 0$ as $X^{2}+Z^{2} \rightarrow \infty[1]$.

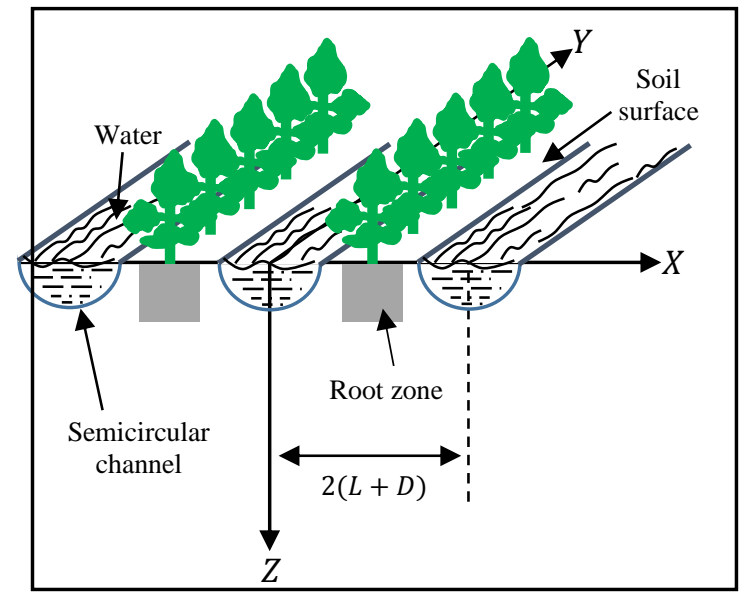

Fig. 1. Geometry of periodic trapezoidal channels with roots zone. 


\section{Basic equation}

The governing equation of the problems in this study that may be used is

$$
\frac{\partial \theta}{\partial t}=\frac{\partial}{\partial X}\left(K \frac{\partial \psi}{\partial X}\right)+\frac{\partial}{\partial Z}\left(K \frac{\partial \psi}{\partial Z}\right)-\frac{\partial K}{\partial Z}-S(X, Z, \psi),
$$

where $K$ is the hydraulic conductivity, $\theta$ is water or moisture content in the soil, $\psi$ is the suction potential, and $S$ is the rootwater uptake function as in $[3,4]$. Function $S$ is defined as

$$
S(X, Z, \psi)=\gamma(\psi) \frac{L_{t} \beta(X, Z) T_{p o t}}{\int_{0}^{Z_{m}} \int_{L+D-X_{m}}^{L+D} \beta(X, Z) d X d Z},
$$

where $L_{t}$ is the width of the soil surface associated with transpiration process, $\beta$ is the spatial root-water uptake distribution, $T_{p o t}$ is the transpiration potential, and is the rootwater stress response function reported by Utset et al [7]. The spatial root-water uptake, $\beta$, is

$$
\beta(X, Z)=\left(1-\frac{L+D-X}{X_{m}}\right)\left(1-\frac{Z}{Z_{m}}\right) e^{-H},
$$

for $L+D-X_{m} \leq X \leq L+D$ and $0 \leq Z \leq Z_{m}$. Here

$$
H=\frac{P_{Z}}{Z_{m}}\left|Z^{*}-Z\right|+\frac{P_{X}}{X_{m}}\left|X^{*}-(L+D-X)\right| .
$$

Using the Kirchhoff transformation

$$
\Theta=\int_{-\infty}^{\psi} K(t) d t
$$

and

$$
K=K_{s} e^{\alpha \psi}
$$

where $K_{s}$ is the saturated hydraulic conductivity and $\alpha$ is the soil parameter, the suction potential can be formulated as

$$
\psi=\frac{1}{\alpha} \ln \left(\frac{\alpha \Theta}{K_{s}}\right),
$$

and (1) can be written as

$$
\frac{1}{D(\theta)} \frac{\partial \Theta}{\partial t}=\frac{\partial^{2} \Theta}{\partial X^{2}}+\frac{\partial^{2} \Theta}{\partial Z^{2}}-\alpha \frac{\partial \Theta}{\partial Z}-S(X, Z, \psi) .
$$

Here $D(\theta)=K d \psi / d \theta$ is the diffusivity. The diffusivity $D$ may be assumed as a constant $d$ in the case of high frequency irrigation [8]. The flux normal pointing out the region with normal vector $\mathbf{n}=\left(n_{1}, n_{2}\right)$ is

$$
F=U n_{1}+V n_{2}
$$

where

$$
U=-\frac{\partial \Theta}{\partial X} ; V=\alpha \Theta-\frac{\partial \Theta}{\partial Z}
$$

Substituting the dimensionless variables

$$
\begin{gathered}
x=\frac{\alpha X}{2} ; z=\frac{\alpha Z}{2} ; \Phi=\frac{\pi \Theta}{v_{0} L} ; u=\frac{2 \pi}{\alpha v_{0} L} U ; \\
v=\frac{2 \pi}{\alpha v_{0} L} V ; f=\frac{2 \pi}{\alpha v_{0} L} F ; t=\frac{\alpha^{2} d}{4} T
\end{gathered}
$$

into (8), yield

$$
\frac{\partial \Phi}{\partial t}=\frac{\partial^{2} \Phi}{\partial x^{2}}+\frac{\partial^{2} \Phi}{\partial z^{2}}-2 \frac{\partial \Phi}{\partial z}-\gamma^{*}(\Phi) \mathrm{s}^{*}(x, z),
$$

where $\gamma^{*}$ and $s^{*}$ are as in [3].

Applying transformation

$$
\Phi(x, z, t)=\Phi^{*}(x, z, t) e^{t},
$$

into (12), yields

$$
\frac{\partial \Phi^{*}}{\partial t}=\frac{\partial^{2} \Phi^{*}}{\partial x^{2}}+\frac{\partial^{2} \Phi^{*}}{\partial z^{2}}-\Phi^{*}-\gamma^{*}(\Phi) \mathrm{s}^{*}(x, z) .
$$

Using the fundamental solution of Laplace equation

$$
\varphi(x, z ; \xi, \eta)=\frac{1}{4 \pi} \ln \left[(x-\xi)^{2}+(y-\eta)^{2}\right]
$$

(14) is recast to integro-differential form

$$
\lambda(\xi, \eta) \Phi^{*}(\xi, \eta, t)
$$

$$
\begin{gathered}
=\iint_{R} \varphi(x, z ; \xi, \eta)\left[\Phi^{*}(x, z, t)+\gamma^{*}(\Phi) s^{*}(x, z) e^{-z}\right. \\
\left.+\frac{\partial \Phi^{*}}{\partial t}\right] d x d z \\
+\int_{C}\left[\Phi^{*}(x, z) \frac{\partial}{\partial n}[\varphi(x, z ; \xi, \eta)]\right. \\
\left.-\varphi(x, z ; \xi, \eta) \frac{\partial}{\partial n}\left[\Phi^{*}(x, z)\right]\right] d s(x, z)
\end{gathered}
$$

where 


$$
\lambda(\xi, \eta)=\left\{\begin{array}{cc}
1 / 2, & (\xi, \eta) \text { on smooth part of } C \\
1, & (\xi, \eta) \in R
\end{array} .\right.
$$

Applying Laplace transform

$$
\phi(x, z, s)=\int_{0}^{\infty} e^{-s t} \Phi^{*}(x, z, t) d t,
$$

subject to the initial condition

$$
\Phi^{*}(x, z, 0)=0,
$$

on (16) yields

$$
\begin{aligned}
& \lambda(\xi, \eta) \phi(\xi, \eta, s)=\iint_{R} \varphi(x, z ; \xi, \eta)[(1+s) \phi(x, z, s) \\
&\left.+\frac{1}{s} \gamma^{*}(\Phi) s^{*}(x, z) e^{-z}\right] d x d z \\
&+\int_{C}\left[\phi(x, z, s) \frac{\partial}{\partial n}[\varphi(x, z ; \xi, \eta)]\right. \\
&\left.-\varphi(x, z ; \xi, \eta) \frac{\partial}{\partial n}[\phi(x, z, s)]\right] d s(x, z),(20)
\end{aligned}
$$

It can be seen that there is a non-linear term on the right hand side of (20), and hence the direct use of the Laplace Transform challenging. To overcome this difficulty, we employ a predictor-corrector scheme. Detailed of the scheme can be obtain in [9].

Integro-differential equation (20) is the integrodifferential equation of equation

$$
\frac{\partial^{2} \phi}{\partial x^{2}}+\frac{\partial^{2} \phi}{\partial z^{2}}=(1+s) \phi+\frac{1}{s} \gamma^{*}(\Phi) s^{*}(x, z) e^{-z}
$$

Boundary conditions of the problem in terms of $\phi$ are

$\frac{\partial \phi}{\partial n}=\frac{2 \pi}{\alpha L} e^{-z}-n_{2} \phi$, on the surface of the channel,

$\frac{\partial \phi}{\partial n}=-\phi$, on the surface of soil outside the channel,

$\frac{\partial \phi}{\partial n}=0, x=0$ and $z \geq 0$,

$\frac{\partial \phi}{\partial n}=0, x=b$ and $z \geq 0$,

$\frac{\partial \phi}{\partial n}=-\phi, 0 \leq x \leq b$ and $z=\infty$.

The term $n_{2}$ in (22) is the vertical component of normal vector pointing out region $R$. After solving (21) subject to boundary conditions (22) to (26), numerical values of $\phi$ may be obtained. These values are then transformed using the Stehfest formula

$$
\Phi^{*}(x, z, t) \simeq \frac{\ln 2}{t} \sum_{p=1}^{2 P} K_{n} \phi\left(x, z, s_{n}\right),
$$

where

$$
\begin{gathered}
s_{n}=n \frac{\ln 2}{t}, \\
\sum_{m=(n+1) / 2}^{K_{n}=(-1)^{n+P} \times} \frac{m^{p}(2 m) !}{(P-m) ! m !(m-1) !(n-m) !(2 m-n) !},
\end{gathered}
$$

and $P$ is a positive integer, is then used to determine the numerical values of their inverse Laplace transforms, which are the dimensionless MFP. In this study, we use $P=3$.

\section{RESUlt AND DISCUSSION}

To apply the method described in the preceding section, we set $L=D=50 \mathrm{~cm}$. The potential transpiration rate, $T_{p o t}$, is $4 \mathrm{~cm} /$ day [10,11]. The values of experimental parameters $\alpha$ and $K_{s}$ of Pima Clay Loam are $0.014 \mathrm{~cm}^{-1}$ and $9.9 \mathrm{~cm} /$ day, respectively. These values are as reported by Amozegar-Fard et al [12] and Bresler [13].

The root zones are assumed to have the same width and depth of $100 \mathrm{~cm}$. Hence we have $X_{m}=50 \mathrm{~cm}$ and $Z_{m}=100$ $\mathrm{cm}$. Two different root uptakes, namely Root A and Root B, are considered. Parameter values for Root A and Root B are summarized in Table 1.

The root-water stress response function used here is identical to that reported by Utset et al [7], which can be seen graphically in Fig. 2. The value of $h_{3}$ for $T_{p o t}=0.4 \mathrm{~cm} / \mathrm{day}$ is interpolated from $h_{3, a}$ and $h_{3, b}$, and we have $h_{3}=-470$.

The LTDRM with a predictor-corrector scheme is employed to obtain numerical solutions to (21) subject to boundary conditions (22) to (26). In order to employ the method, the domain must be bounded by a simple closed curve. An appropriate depth for boundary conditions to be applied without significant impact to values of $\phi$ in the domain is $z=4$. Therefore, the domain is set to be between $z$ $=0$ and $z=4$. The boundary is discretized into 402 constant elements, and 894 interior points are chosen. Some of the results are presented in Table 2 to Table 5.

Table 1. Fitting Parameters for Root A and Root B

\begin{tabular}{|l|c|c|c|c|}
\hline \multirow{2}{*}{\multicolumn{1}{|c|}{ Type of Root }} & \multicolumn{4}{|c|}{ Parameters } \\
\cline { 2 - 5 } & $\boldsymbol{X}^{*}$ & $\boldsymbol{Z}^{*}$ & $\boldsymbol{P}_{\boldsymbol{X}}$ & $\boldsymbol{P}_{\boldsymbol{Z}}$ \\
\hline Root A & $0 \mathrm{~cm}$ & $0 \mathrm{~cm}$ & 1.0 & 1.0 \\
\hline Root B & $20 \mathrm{~cm}$ & $0 \mathrm{~cm}$ & 1.0 & 1.0 \\
\hline
\end{tabular}




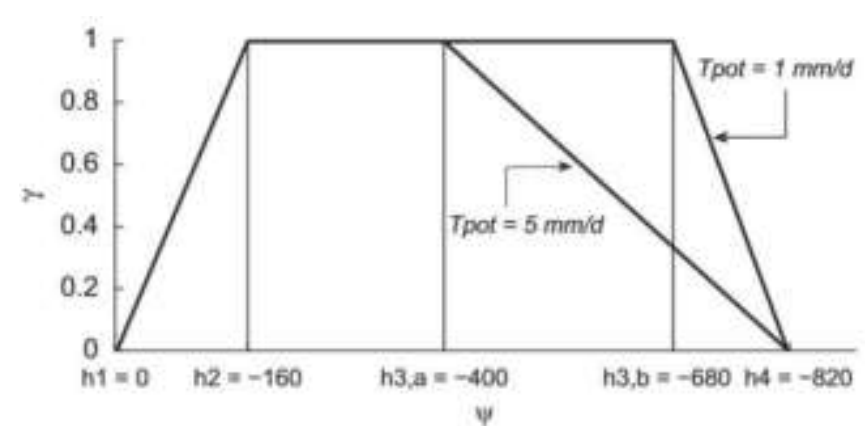

Fig. 2. Graph of root-water stress response function reported by Utset et al.

Table 2. Values of $S$ at $(60 \mathrm{~cm}, 40 \mathrm{~cm})$

\begin{tabular}{|c|c|c|}
\hline \multicolumn{3}{|c|}{ Root-water uptake $(\boldsymbol{S})$} \\
\hline $\boldsymbol{t}$ & Root $\boldsymbol{A}$ & Root $\boldsymbol{B}$ \\
\hline 0.8 & 0.000597 & 0.000651 \\
\hline 1.0 & 0.000575 & 0.000627 \\
\hline 2.0 & 0.000536 & 0.000584 \\
\hline 3.0 & 0.000528 & 0.000576 \\
\hline 4.0 & 0.000526 & 0.000574 \\
\hline 5.0 & 0.000526 & 0.000573 \\
\hline
\end{tabular}

Table 3. Values of $S$ at $(60 \mathrm{~cm}, 90 \mathrm{~cm})$

\begin{tabular}{|c|c|c|}
\hline \multicolumn{3}{|c|}{ Root-water uptake $(\boldsymbol{S})$} \\
\hline $\boldsymbol{t}$ & Root $\boldsymbol{A}$ & Root $\boldsymbol{B}$ \\
\hline 0.8 & 0.000061 & 0.000067 \\
\hline 1.0 & 0.000058 & 0.000063 \\
\hline 2.0 & 0.000051 & 0.000056 \\
\hline 3.0 & 0.000050 & 0.000055 \\
\hline 4.0 & 0.000050 & 0.000054 \\
\hline 5.0 & 0.000050 & 0.000054 \\
\hline
\end{tabular}

Table 4. Values of $S$ at $(90 \mathrm{~cm}, 40 \mathrm{~cm})$

\begin{tabular}{|c|c|c|}
\hline \multicolumn{3}{|c|}{ Root-water uptake $(\boldsymbol{S})$} \\
\hline $\boldsymbol{t}$ & Root $\boldsymbol{A}$ & Root $\boldsymbol{B}$ \\
\hline 0.8 & 0.004944 & 0.005390 \\
\hline 1.0 & 0.004750 & 0.005180 \\
\hline 2.0 & 0.004416 & 0.004816 \\
\hline 3.0 & 0.004350 & 0.004743 \\
\hline 4.0 & 0.004331 & 0.004723 \\
\hline 5.0 & 0.004326 & 0.004717 \\
\hline
\end{tabular}

Table 5. Values of $S$ at $(90 \mathrm{~cm}, 90 \mathrm{~cm})$

\begin{tabular}{|c|c|c|}
\hline \multicolumn{3}{|c|}{ Root-water uptake $(\boldsymbol{S})$} \\
\hline $\boldsymbol{t}$ & Root $\boldsymbol{A}$ & Root $\boldsymbol{B}$ \\
\hline 0.8 & 0.000465 & 0.000507 \\
\hline 1.0 & 0.000438 & 0.000477 \\
\hline
\end{tabular}

\begin{tabular}{|c|c|c|}
\hline \multicolumn{3}{|c|}{ Root-water uptake $(\boldsymbol{S})$} \\
\hline $\boldsymbol{t}$ & $\boldsymbol{R o o t} \boldsymbol{A}$ & Root $\boldsymbol{B}$ \\
\hline 2.0 & 0.000390 & 0.000426 \\
\hline 3.0 & 0.000380 & 0.000415 \\
\hline 4.0 & 0.000378 & 0.000412 \\
\hline 5.0 & 0.000377 & 0.000411 \\
\hline
\end{tabular}

Table 2 to Table 5 show the values of $S$ at different points. Specifically, Table 2 shows $S$ at $(60 \mathrm{~cm}, 40 \mathrm{~cm})$. Values of $S$ at $(60 \mathrm{~cm}, 90 \mathrm{~cm})$ are shown in Table 3. Table 4 and Table 5 show $\mathrm{S}$ at $(90 \mathrm{~cm}, 40 \mathrm{~cm})$ and $(90 \mathrm{~cm}, 90 \mathrm{~cm})$ respectively. It can be seen that the values of $S$ decrease as $t$ increase. These results indicate that suction potential, $\psi$, increases as $t$ increases, as values of $\psi$ in this study are between -160 and 0 . It can also be seen that the percentage of decrease in $S$ get smaller as $t$ increases, and the change in $S$ from $t=4$ to $t=5$ is almost $0 \%$. Hence, at $t=5$ values of $S$ almost reach their steady state values.

From the tables above, values of $\mathrm{S}$ at $X=90 \mathrm{~cm}$ are much higher than those at $X=60 \mathrm{~cm}$. These results show that highest uptake is at location near the plant, and the lowest uptake is at that further. We also observe that there is a rapid drop in the values of $S$ about $91 \%$ when comparing the values of $S$ at $Z=40 \mathrm{~cm}$ and $Z=90 \mathrm{~cm}$. Hence, the uptake at shallower locations are much higher than those deeper.

\section{CONCLUSION}

A problem involving time dependent infiltration from periodic semicircular channels with root-water uptake has been solved numerically using an LTDRM and a predictorcorrector scheme simultaneously. We first transform the governing equation of the infiltration problem into a modified Helmholtz equation. After solving the modified Helmoltz equation numerically, the solutions are then transformed back using Stehfest formula to obtain solutions of the governing equation. Using these solutions, values of root-water uptake function can be obtained. The results indicate that as $t$ increases, the uptake decreases and reach a steady state value at some value of $t$. The results show that maximum uptake is at location near the plant, and the minimum uptake is at that further.

\section{ACKNOWLEDGMENT}

This research was supported by Research Grant Department of Mathematics UGM-RG contract number 0130/J01.1.28/PL.06.02/2017

\section{REFERENCES}

[1] V. Batu, "Steady infiltration from single and periodic strip sources," Soil Science Society of America Journal, vol. 42, pp. 544-549, 1978.

[2] D. L. Clements, N. Widana, and M. Lobo, "A hypersingular boundary integral equation for a class of problems concerning infiltration from periodic channels," Electronic Journal of Boundary Elements, vol. 5, pp. 1-16, 2007.

[3] I. Solekhudin, "Water infiltration from periodic trapezoidal channels with different types of root-water uptake," Far East Journal of Mathematical Sciences, vol. 100, pp. 2029-2040, 2016. 
[4] I. Solekhudin, "Suction potential and water absorption from periodic channels in a homogeneous soil with different root uptake," Advances and Applications in Fluid Mechanics, vol. 20, pp. 127-139, 2017.

[5] D. L. Clements and M. Lobo, "A BEM for time dependent infiltration from an irrigation channel," Engineering Analysis with Boundary Elements, vol. 34, pp. 1100-1104, 2010.

[6] I. Solekhudin and Sumardi, "A DRBEM for steady infiltration from periodic semi-circular channels with two different types of roots distribution," AIP Conference Proceedings 1848, 040009-1-0400097, 2017.

[7] A. Utset, M. E. Ruiz, J. Garcia, and R. A. Feddes, "A SWARCOPbased potato root water-uptake function as determined under tropical conditions," Potato Res., vol. 43, pp. 19-29, 2000.

[8] H. A. Basha, "Multidimensional linearized nonsteady infiltration with prescribed boundary conditions at the soil surface," Water Resource Research, vol. 35, pp. 75-83, 1999.
[9] I. Solekhudin and K. C. Ang, "A Laplace transform DRBEM with a predictor-corrector scheme for time dependent infiltration from periodic channels with root-water uptake," Engineering Analysis with Boundary Elements, vol. 50, pp. 141-147, 2015.

[10] K. Y. Li, R. de Jong, and J. B. Boisvert, "An exponential root-water uptake model with water stress compensation," J. of Hydrol., vol. 252, pp. 189-204, 2001.

[11] J. Šimunek and J. W. Hopmans, "Modeling compensated root water and nutrient uptake," Ecol. Model, vol. 220, pp. 505-521, 2009.

[12] A. Amoozegar-Fard, A. W. Warrick, and D. O. Lomen, "Design nomographs for trickle irrigation systems," J. of Irrigation and Drainage Engineering, vol. 110, pp. 107-120, 1984.

[13] E. Bresler, "Analysis of trickle irrigation with application to design problems," Irrigation Science vol. 1, pp. 3-17, 1978. 